\title{
Understanding Quality Assessment in Research and Meta-Analysis
}

\author{
Dhananjoy Shaw ${ }^{1 *}$, Syed Murtaza Hussain Andrabi ${ }^{2}$ and Deepak \\ Singh $^{3}$ \\ ${ }^{1}$ Officiating Principal, Indira Gandhi Institute of Physical Education and Sports \\ Sciences, University of Delhi, New Delhi, India \\ ${ }^{2}$ PhD Student, Department of Physical education and Sports science, University \\ of Delhi, New Delhi, India \\ ${ }^{3}$ Masters Degree Student, IGIPESS, University of Delhi, New Delhi, India \\ *Corresponding Author: Dhananjoy Shaw, Officiating Principal, Indira Gandhi \\ Institute of Physical Education and Sports Sciences, University of Delhi, New Delhi, \\ India.
}

In academia, research is utilized as an indispensable tool for either discovering or inventing new knowledge or modifying the current knowledge of the academic discipline. Numerous types of research designs are used in the academic research and each design has its own advantages and disadvantages [1]. How much a particular research design contributes to the ultimate aim of research (i.e. finding new knowledge or modifying the current knowledge) determines the superiority of that research design in comparison to others. A technique that ranks the research designs based on their contribution to the advancement of knowledge or the modification of the current knowledge is known as the hierarchy of evidence. Hierarchy of evidence is defined as a technique to ranks the study or research designs on the basis of the relative strength of their results or evidence $[2,3]$.

Meta-analysis is the most prolific research design because it is an "analysis of analyses" which means that it combines and analyse the results from a number of independent research studies or analyses on a particular area or research problem and then synthesizes collective conclusions in regard to that area or research problem $[4,5]$.

The quality of a meta-analysis is only as good as the quality of the independent research studies that it endorses. A major threat to the quality of the research studies and consequently, the quality of the meta-analysis is "bias". Bias is any intentional or unintentional deviation from the truth in data collection, data analysis, interpretation and publication of research study [6,7]. Biases
Received: August 10, 2020

Published: October 17, 2020

(C) All rights are reserved by Dhananjoy

Shaw., et al. lead to false conclusions in the research studies and if such studies are included in a meta-analysis then it jeopardizes the validity and quality of the meta-analysis. Broadly speaking, biases in research can come from four major areas.

\section{Bias in data collection}

Bias in data collection exists due to the practice of using a representative sample from a population to study a phenomenon rather than studying the whole population. Most common type of bias in data collection is "selection bias". Selection bias occurs when a certain set of individuals are more likely to be selected as the subject of the study rather than other individuals from the same population. This causes an over-representation of selected set of individuals and underrepresentation of other individuals who are less likely to be selected in the sample which then compromises representativeness of the sample for the population. This compromises the "truth" of the conclusions of the study for population and generates "bias". Bias in data collection can also occur if sample size is too sample to represent the population [7].

\section{Bias in data analysis}

Bias in data analysis exists due to the predisposition in the researchers to favour statistically significantly results which favour their research hypothesis. Bias in data analysis occurs when the researchers performs data fabrication, abusing of data in terms of eliminating data which don't support hypothesis, use of inappropriate statistical tests and $\mathrm{P}$ value hunting. $\mathrm{P}$ value hunting means that the researcher performs unnecessary multiple pair wise com- 
parisons and further divides the data into sub group to perform secondary or sub group analysis to find such result with a $P$ value which makes their result significant irrespective of the hypothesis. This compromises the "truth" of the conclusions of the study and thus, generates "bias" $[6,7]$.

\section{Bias in data interpretation}

Bias in data interpretation exists due to the tendency in researcher to hold on to their hypothesis regardless of the results of the study. This bias occurs when the researchers believe in their hypothesis take precedence over the results of their study which causes them to neglect the results by interpreting them in the favour of their hypothesis. This is also a bias because it causes the interpretation of the result to deviate from the actual "truth" of the results which can mislead the readers especially novice researchers $[8,9]$.

\section{Publication bias}

Publication bias exists because of the tendency of the research journals and publishers to favour the publication of a study with positive and significant results over a study with negative and non-significant results regarding a phenomenon. This bias directly threatens the validity of a meta-analysis because only studies with positive and significant results will be included in the meta-analysis and thus, the findings of such meta-analysis will deviate from the actual "truth" of the concerned phenomenon because the negative and non-significant results will be underrepresented or absent in the findings $[6,9,10]$.

\section{Tools to assess the quality of research}

Unfortunately, biases are rampantly present in the research literature and this puts serious threats to the validity of the metaanalysis. To tackle this problem, the researchers had developed a method which acts a filter to identify and remove the studies with biases before they are included in the meta-analysis and this method is known as "quality assessment". Here is an account of few methods that are commonly employed.

\section{Cochrane manual}

Cochrane is a British international charitable organisation which organises medical research findings [11]. Cochrane has developed it's official guide known as Cochrane Handbook for Systematic Reviews of Interventions. Cochrane's official guide has provided a tool for assessing risk for bias and that tool is known as “The Cochrane Collaboration's tool for assessing risk of bias" [12].

\section{PRISMA guidelines}

PRISMA stands for Preferred Reporting Items for Systematic reviews and Meta-Analyses. PRISMA is a method used for systematically writing and reporting systematic reviews and meta- analysis. PRISMA has issued a document called PRISMA statement which has PRISMA Checklist to assess the risk of bias. PRISMA checklist is 27 item checklist that helps to assess risk of bias [13].

\section{JADAD or oxford quality scoring system}

This method was a seminal work by Alejandro R. Jadad and is named after him and is sometimes called Oxford Quality Scoring System. This method is meant for non-randomized studies and has an 8 item list that are put against the purposeful article to quantitatively test its assessment. It has four more items in the additional criteria for comparative studies. This scoring system is frequently used in medical science studies. This grading system is often regarded as flawed and over simplistic [9,14].

\section{MINORS (methodological index of non-random studies)}

MINORS is a valid tool that was designed to assess the methodological quality of non-randomized surgical studies for both comparative and non-comparative studies. This method was developed by a group of scientists in which a list of 12 items was send to 100 experts with surgical specialties for evaluation. This method is suitable for assessing the quality of non-randomized surgical studies [10].

\section{Concluding Remarks}

This article discussed briefly the tools that are predominantly used in meta-analysis and review studies across various disciplines in academia to aid the quality assessment process. However enough thought should be given when employing one tool or the other. This is so because various tools available are competent to assess quality of article in on domain or the other. E.g. MINORS is a valid tool to assess the quality of non-random surgical studies unless it is explicitly stated that they can be used for other studies in their manual, applying this format in studies other than surgical studies would make this method incompetent.

Cochrane manual is used in the meta-analysis and review studies that are to be published in the journal under Cochrane library and hence it has a limited scope of use. However, PRISMA guidelines are open to be used across various disciplines, but it has one of the most tedious procedures. On the other hand MINORS and JADAD are specific to medical sciences, and should not be employed outside this discipline.

Citation: Dhananjoy Shaw., et al. “Understanding Quality Assessment in Research and Meta-Analysis”. Acta Scientific Medical Sciences 4.11 (2020): $52-54$. 


\section{Bibliography}

1. Organizing Academic Research Papers: Types of Research Designs.

2. Hierarchy of evidence.

3. http://www.betsiresearch.wales.nhs.uk/sitesplus/documents/836/Hierarchy\%20of\%20 evidence $\% 20$ factsheet $\% 20$ v1\%2011042016.pdf

4. Evidence-Based Dentistry: Determining Strength of Evidence.

5. Evidence-Based Practice: Study Design.

6. Meta-analysis course: part 1: Systematic reviews, meta-analysis, and introduction to $\mathrm{R}$.

7. Šimundić AM. "Bias in research". Biochemia Medica 23.1 (2013): 12-15.

8. Dreier M. "Quality Assessment in Meta-analysis". Methods of Clinical Epidemiology (2013): 213-228.

9. Jadad AR., et al. "Assessing the Quality of Reports of Randomized Clinical Trials: Is Blinding Necessary?" Controlled Clinical Trials 12 (1995): 1-12.

10. Slim K., et al. "Methodological index for non-randomized studies (MINORS): development and validation of a new instrument". ANZ Journal of Surgery 73.9 (2003): 712-716.

11. Cochrane (organisation).

12. Table 8.5.a: The Cochrane Collaboration's tool for assessing risk of bias.

13. http://www.prisma-statement.org/\#: :text=PRISMA $\% 20$ is $\% 20$ an $\% 20$ evidence $\% 2$ Dbased,research $\% 2$ C $\% 20$ particularly\%20 evaluations $\% 20$ of\%20interventions

14. http://www.pmidcalc.org/?sid=8721797\&newtest $=Y$

\section{Assets from publication with us}

- Prompt Acknowledgement after receiving the article

- Thorough Double blinded peer review

- Rapid Publication

- Issue of Publication Certificate

- High visibility of your Published work

Website: www.actascientific.com/

Submit Article: www.actascientific.com/submission.php

Email us: editor@actascientific.com

Contact us: +919182824667 